\title{
Gravitational waves from binary black hole mergers surrounded by scalar field clouds: Numerical simulations and observational implications
}

\author{
Sunil Choudhary, ${ }^{1}$ Nicolas Sanchis-Gual, ${ }^{2}$ Anshu Gupta, ${ }^{1}$ \\ Juan Carlos Degollado, ${ }^{3}$ Sukanta Bose, ${ }^{1,4}$ and José A. Font ${ }^{5,6}$ \\ ${ }^{1}$ Inter-University Centre for Astronomy and Astrophysics, Post Bag 4, Ganeshkhind, Pune 411 007, India \\ ${ }^{2}$ Centro de Astrofísica e Gravitação - CENTRA, \\ Departamento de Física, Instituto Superior Técnico - IST, \\ Universidade de Lisboa - UL, Avenida Rovisco Pais 1, 1049-001, Portugal \\ ${ }^{3}$ Instituto de Ciencias Físicas, Universidad Nacional Autónoma de México, \\ México Apdo. Postal 48-3, 62251, Cuernavaca, Morelos, México \\ ${ }^{4}$ Department of Physics \&3 Astronomy, Washington State University, 1245 Webster, Pullman, WA 99164-2814, U.S.A. \\ ${ }^{5}$ Departamento de Astronomía y Astrofísica, Dr. Moliner 50, 46100, Burjassot (València), Spain \\ ${ }^{6}$ Observatori Astronòmic, Universitat de València, \\ C/ Catedrático José Beltrán 2, 46980, Paterna (València), Spain
}

\begin{abstract}
We show how gravitational-wave observations of binary black hole (BBH) mergers can constrain the physical characteristics of a scalar field cloud parameterized by mass $\tilde{\mu}$ and strength $\phi_{0}$ that may surround them. We numerically study the inspiraling equal-mass, non-spinning BBH systems dressed in such clouds, focusing especially on the gravitational-wave signals emitted by their mergerringdown phase. These waveforms clearly reveal that larger values of $\tilde{\mu}$ or $\phi_{0}$ cause bigger changes in the amplitude and frequency of the scalar-field-BBH ringdown signals. We show that the numerical waveforms of scalar-field-BBHs can be modelled as chirping sine-Gaussians, with matches in excess of $95 \%$. This observation enables one to employ computationally expensive Bayesian studies for estimating the parameters of such binaries. Using our chirping sine-Gaussian signal model we establish that observations of $\mathrm{BBH}$ mergers at a distance of $450 \mathrm{Mpc}$ will allow to distinguish $\mathrm{BBHs}$ without any scalar field from those with a field strength $\phi_{0} \gtrsim 5.5 \times 10^{-3}$, at any fixed value of $\tilde{\mu} \in[0.3,0.8]$, with $90 \%$ confidence or better, in single detectors with Advanced LIGO/Virgo type sensitivities. This provides hope for the possibility of determining or constraining the mass of ultra-light bosons with gravitational-wave observations of $\mathrm{BBH}$ mergers. [This manuscript has been assigned the preprint number LIGO-2000375]
\end{abstract}

\section{INTRODUCTION}

The gravitational-wave (GW) detector network comprising the Advanced LIGO (aLIGO) and the Advanced Virgo (AdV) interferometers recently launched the era of GW astronomy. The landmark observation in 2015 of a GW signal from a binary black hole $(\mathrm{BBH})$ merger and the subsequent additional detections of binary mergers that followed during the first two observational campaigns (O1 and O2), including a binary neutron star (BNS) system [1], have put GW astronomy on a very firm footing. Moreover, during O3 GW candidate events have been released as public alerts to facilitate the rapid identification of electromagnetic or neutrino counterparts, expanding the capabilities of multi-messenger astronomy. A significant number of candidates have been publicly announced on the GW candidate event database ${ }^{1}$ and some confirmed detections have already been published [2-6].

Accurate computations of the gravitational waveform of a compact binary coalescence event, especially in the early inspiralling stage, yield a plethora of information about the binary and the physics of its components. While large banks of waveforms are available for BBHs, and also for BNS mergers and BH-NS systems, relatively

\footnotetext{
${ }^{1}$ gracedb.ligo.org
}

less information is available about possible departures from those waveforms if the binary components were exotic (yet physically plausible) compact objects, e.g. boson stars, Proca stars, gravastars, fuzzballs or wormholes. However, there are ongoing theoretical efforts to investigate them [7-12]. In particular, the merger of binary compact objects formed by fundamental bosonic fields has been explored in several works, including head-on collisions and orbital mergers of boson stars, oscillatons, and Proca stars [13-23], The potential of GW astronomy for new discoveries might eventually shed light on the actual existence in Nature of those theoretical proposals.

Using GW observations as probes of new physics is challenging but they also provide a brand new experimental channel to try and find answers to the biggest unsolved problems in fundamental physics (see, e.g. [24]). Some of those are the nature of dark matter and dark energy, the physics in the early Universe, and possible extensions of the Standard Model. A well-known example for physics beyond the Standard Model is provided by ultra-light bosonic particles. The masses of the ultralight bosons of the string axiverse can range from $10^{-33}$ $\mathrm{eV}$ to $10^{-10} \mathrm{eV}$ [25]. Even though their masses can be smaller than those of known particles, their existence is possible if the coupling to ordinary matter is very weak.

In particular scalar fields surrounding supermassive $\mathrm{BHs}$ in galactic centers have been proposed as candidates 
for dark matter [26-30]. This model assumes that dark matter is composed of bosonic particles that may condensate into macroscopic objects around BHs. The justification for this proposal requires long-lived scalar-field configurations. Their dynamics and lifetime have been studied both in the linear regime [31-35] and with non-linear simulations in general relativity [36-38], providing convincing support to the proposal. Specifically, the early work by [33] in the linearized regime showed that massive scalar fields surrounding stationary and non-rotating BHs could indeed form such quasi-bound states as a result of the presence of a potential well due to the mass term. These states decay at infinity and are characterized by a complex frequency whose real part represents the actual oscillation frequency while the imaginary part gives, depending on the sign, either their decay rate or the growth rate, if a mode is superradiantly unstable.

The superradiant instability operates in rotating black holes (but see [39] for an academic setup with a ReissnerNordström BH) where bosonic waves scattered off the BH extract energy and angular momentum and increase the energy of the field through the classical process of superradiance [40, 41] (see also [42] and references therein). The nonlinear realisation of superradiance in Kerr BHs was recently shown by $[43,44]$ employing a vector (Proca) bosonic field (see also $[45,46]$ ). The end-state of this process is the formation of hairy BHs, i.e. Kerr BHs surrounded by either scalar or vector hair, in which the bosonic field is in equilibrium (i.e. synchronized) with the BH $[47,48]$. Recent works [49-58] have investigated possible observational signatures of the bosonic clouds through the detection of the nearly monochromatic GWs they emit, providing procedures to e.g. constrain the QCD axion [49], probe ultra-light bosons in BBH inspirals through the analysis of resonant transitions between growing and decaying modes of the clouds [53] and estimate upper limits for the detectability of ultra-light bosons through direct GW searches [50, 52]. Recent attempts [58] promote the use of third-generation groundbased GW detectors combined with the spaced-based LISA mission to increase the chances of detection using a multiband technique. We also note that the direct detection of bosonic fields in the form of bosonic stars has been recently proposed in connection with GW190521 [59]. Lastly, for its relevance to our setup and results, we highlight the work of [51] which investigated the joint evolution of intermediate-mass BBH surrounded by a shell of an axion-like scalar field of different strenghts, finding that the dynamics of the mergers can be modified by the presence of the environmental scalar field cloud, which also impacts the GW emission.

In this work we also investigate if $\mathrm{GW}$ measurements can probe the existence of bosonic clouds around BHs but we employ a different setup to that used in most previous works. We study if the presence of a scalar field cloud might actually be established through its imprint on the GWs from BBH mergers. Our goal is to show through a combination of numerical-relativity sim- ulations and Bayesian inference if the actual network of GW interferometers can measure the differences in the waveforms induced by the presence of scalar field clouds around the coalescing BHs. To this aim we parameterize the cloud by its mass $\tilde{\mu}$ and its strength $\phi_{0}$. Our investigation reveals that it may actually be possible to observationally distinguish $\mathrm{BBHs}$ without any scalar field from those with a field strength of order $\phi_{0} \gtrsim 5.5 \times 10^{-3}$, at any fixed value of $\tilde{\mu} \in[0.3,0.8]$, with $90 \%$ confidence or better, in single detectors with aLIGO or AdV type sensitivity, up to distances of about $450 \mathrm{Mpc}$. At smaller distances ( 100-200 Mpc) even less strong fields might be distinguishable.

This paper is organized as follows: In section II we briefly summarise our basic framework to study the dynamics of $\mathrm{BBH}$ mergers endowed with bosonic clouds. Section III describes the numerical setup, the initial data, and the results of the numerical simulations for varying scalar-field parameters. The measurement and estimation of these parameters through Bayesian inference are discussed in Section IV and we close with Section V which presents our conclusions. Appendix B provides specific details on our numerical waveforms. Throughout the paper we use natural units, $c=G=\hbar=1$.

\section{BASIC FRAMEWORK}

Our approach to model BBH mergers surrounded by a scalar field environment considers a massive complex scalar field interacting through gravity with the BHs. The system is governed by the Einstein-Klein-Gordon theory. Details of the formulation have been given in $[36,60]$ which we briefly summarise here. We consider a complex scalar field $\Phi$ minimally coupled to gravity described by the action

$S=\int d^{4} x \sqrt{-g}\left(\frac{1}{16 \pi} R-\frac{1}{2} g^{\alpha \beta} \nabla_{\alpha} \Phi^{*} \nabla_{\beta} \Phi-\frac{1}{2} m_{s}^{2} \Phi \Phi^{*}\right)$,

where $R$ is the Ricci scalar associated with the metric $g_{\alpha \beta}$, with determinant $g$, and $m_{s}$ is the mass of the field. The asterisk symbol indicates the complex conjugate operation and $\nabla_{\alpha}$ denotes the covariant derivative. Minimizing this action with respect to the metric and with respect to the scalar field yields the Einstein-KleinGordon system:

$$
R_{\alpha \beta}-\frac{1}{2} g_{\alpha \beta} R=8 \pi T_{\alpha \beta},
$$

and

$$
g^{\alpha \beta} \nabla_{\alpha} \nabla_{\beta} \Phi=m_{s}^{2} \Phi,
$$

where $T_{\alpha \beta}$ is the stress-energy tensor

$$
\begin{aligned}
T_{\alpha \beta}= & \frac{1}{2}\left(\nabla_{\alpha} \Phi \nabla_{\beta} \Phi^{*}+\nabla_{\alpha} \Phi^{*} \nabla_{\beta} \Phi\right) \\
& -\frac{1}{2} g_{\alpha \beta}\left(g^{\gamma \sigma} \nabla_{\gamma} \Phi \nabla_{\sigma} \Phi^{*}+m_{s}^{2} \Phi \Phi^{*}\right) .
\end{aligned}
$$


In this set up, the self-gravitating scalar field interacts with the binary through gravity by means of the spacetime metric described by Einstein equations.

Given the total ADM mass $M$ of a gravitational system, we define the dimensionless parameter

$$
\tilde{\mu} \equiv \frac{G M m_{s}}{\hbar c}
$$

to characterize the scalar cloud. This parameter is the ratio of the gravitational radius of the system $R_{g}=G M / c^{2}$, and the Compton wavelength of the scalar field $\lambda_{c}=$ $\hbar /\left(m_{s} c\right)$.

The linear dynamics of scalar fields propagating on a single, non-rotating $\mathrm{BH}$ background has been described in [61]. It was found that regular scalar field configurations in the form of quasi-bound states around Schwarzschild BHs may survive in the vicinity of the compact objects for a certain range of values of the scalar field and BH masses. A detailed analysis of the scalar field configurations including the spectrum of quasi-bound states can be found in [62].

The description of the scalar field assumes a harmonic time dependence, $\Phi(t, \vec{r})=\phi(\vec{r}) e^{-i \omega t}$, with $\omega$ a complex number whose real part indicates the oscillating frequency and whose imaginary part determines the decay rate of the field. For small values of the dimensionless parameter $\tilde{\mu}$ it was found that the decay rate of the quasibound states decreases as a power law of $\tilde{\mu}$. The mass spectrum of axion-like particles that could be probed for a given coupling $\bar{\mu}$ is continuous, and includes, among others, the QCD axion and a large range of particles beyond the Standard Model predicted in the string axiverse scenario [25]. We highlight in particular two regimes with astrophysical relevance for the combination of the scalar field and $\mathrm{BH}$ masses for which the scalar field configurations may live around the central object for longer times than the age of the Universe. The first one occurs when the scalar field mass is of the order of $1 \mathrm{eV}$ and the $\mathrm{BH}$ has a mass smaller that $10^{-17} M_{\odot}$. The second regime corresponds to an ultra-light scalar field with mass smaller than $10^{-22} \mathrm{eV}$ and to a supermassive $\mathrm{BH}$ with mass smaller than $5 \times 10^{10} M_{\odot}$. These scenarios correspond, respectively, to axion distributions of a scalar field around primordial BHs and dark matter halos around supermassive $\mathrm{BHs}$ in the centers of galaxies [63]. For stellar size BHs as the ones employed in this work with mass $\sim 40 M_{\odot}$, the mass of the particle corresponds to $\sim 10^{-12} \mathrm{eV}$

\section{NUMERICAL SIMULATIONS}

In order to write Eqs. (2) and (3) as an evolution system suitable for numerical integration we formulate the Einstein-Klein-Gordon system using the BSSN formulation [64-66] (see also [67, 68]). Our numerical simulations are performed using the open source EINSTEINTOOLKIT infrastructure [69-71]. In addition, the CARPET package $[72,73]$ is used for mesh-refinement capabilities, $\mathrm{BH}$ apparent horizons are found using AHFINDERDIRECT $[74,75]$, and the left-hand-side of the Einstein equations is solved using the MACLACHLAN code [76]. The scalar-field evolution code is our own modification of the the publicly available PROCA thorn from the CANUdA library $[77,78]$ to evolve complex scalar fields. This code has been recently employed to study the stability of spinning bosonic stars [79, 80]. The method-of-lines with a fourth-order Runge-Kutta scheme is employed to integrate the time-dependent differential equations.

\section{A. Initial data}

To set initial data suitable for numerical evolution using the "moving punctures" technique we take advantage of the Bowen-York construction for two BHs in vacuum [81]. A nontrivial analytic solution of the momentum constraint equation, thus, can be found [82] and the Hamiltonian constraint equation can be solved using the puncture approach [83-85]. Once the Hamiltonian and momentum constraints are solved we introduce a nonzero scalar field distribution. The addition of the scalar field to the binary system introduces violations of the constraints (see details in Appendix A). However, our results regarding GW emission show that the initial violation produces only a weak spurious GW signal as long as the amplitude of the scalar field $\phi_{0} \ll 1$ for $\tilde{\mu} \approx 1.0$.

In our study we mainly focus on the post-merger characteristics of the gravitational waveforms. Therefore, for simplicity, we initiate our simulations when the two BHs are in their last orbit prior to merger using initial data for a quasi-circular orbit $[86,87]$. The BHs are positioned at $(x, y, z)=(-1.168 M, 0,0)$ and $(1.168 M, 0,0)$, having linear momentum vectors $(0,-0.333 M, 0)$ and $(0,0.333 M, 0)$.

Our simulations are performed for a non-spinning, equal mass $\left(m_{1}=m_{2}=0.453 M\right) \mathrm{BBH}$ system which is surrounded by a scalar field cloud initially shaped in the form of a Gaussian distribution, $\Phi=\phi_{0} e^{-\left(r-r_{0}\right)^{2} / \lambda^{2}}$ centered at radius $r_{0}=0$, where $\phi_{0}$ is the initial amplitude and $\lambda$ is its half width. We simulate a series of configurations varying the amplitude $\phi_{0}$ between $1.0 \times 10^{-5}$ to $1.0 \times 10^{-2}$ and employing a dimensionless scalar field mass parameter $\tilde{\mu}<1.0$. We have observed that due to the choice of constraint-violating conditions caused by the presence of the cloud numerical inaccuracies dominate the evolution of the system for values of $\tilde{\mu}$ of order $10^{-2}$. Therefore, such values are not considered. On the other hand, high-amplitude fields $\left(\phi_{0} \approx 0.01\right)$ trigger their collapse inside the horizon while very low-amplitude fields $\left(\phi_{0}<5.0 \times 10^{-4}\right)$ lead to evolutions that are almost indistinguishable from the pure vacuum $\mathrm{BBH}$ case. Keeping this in mind, we simulate a fiducial number of 18 configurations, setting the scalar field mass parameter $\tilde{\mu}$ in the range from 0.3 to 0.8 for three scalar field ampli- 
tudes, namely $\phi_{0}=3.5 \times 10^{-3}, 4.5 \times 10^{-3}$, and $5.5 \times 10^{-3}$. These simulations are compared with the vacuum $\mathrm{BBH}$ merger case in the absence of any scalar field.

The mass of the cloud is kept sufficiently small, compared to the total $\mathrm{BBH}$ mass, to ensure that the violation of the constraints does not represent a major drawback of our initial data. For rotating BHs there is a mechanism (superradiance) that allows the cloud to grow up to about $10 \%$ of the mass of the $\mathrm{BH}$. We take this value as an upper bound for the mass of the cloud (which scales quadratically with $\phi_{0}$ ) assuming there is no other mechanism to grow the cloud. In addition we choose $\lambda=15$ which yields a size for the scalar cloud comparable to the physical size of the BHs. Much larger values of $\lambda$ correspond to cloud masses that would result in significant constraint violation in our numerical evolution. Much smaller values (that are still larger than the gravitational radius of the system) would require large field amplitudes to leave any noticeable imprint in the merger waveforms. The effect of varying $\lambda$, along with the contribution of the inspiral part of the GW signal in our results, will be explored in more detail in a future work.

The numerical evolutions are performed on a Cartesian grid with a domain size of $(-320 M, 320 M)$ for all three dimensions. However, we apply reflection symmetry in the $z$ direction; thus, the computational domain in that direction is $(0,320 M)$. The numerical grid has 9 refinement levels, starting with two centers located at each puncture, and with resolution $\{(320,160,80,40$, $20,5,2.5,1.25,0.625) M,(8,4,2,1,0.5,0.25,0.125$, $0.0625) M\}$. The first set of numbers indicates the spatial domain of each level and the second set indicates the resolution.

\section{B. Results of the numerical evolutions}

Our goal is to highlight possible imprints of the presence of scalar field clouds in the gravitational waveforms produced during a BBH merger. GW signals are obtained from the simulations by computing the NewmanPenrose scalar $\Psi_{4}$ defined in terms of the Weyl tensor $C_{\alpha \beta \gamma \delta}[88]$ as

$$
\Psi_{4}=C_{\alpha \beta \gamma \delta} k^{\alpha} \bar{m}^{\beta} k^{\gamma} \bar{m}^{\delta},
$$

where $k$ and $\bar{m}$ are two components of the null tetrad $l, k$, $m, \bar{m}$, that satisfy $\ell \cdot k=-1$ and $m \cdot \bar{m}=1$, and all other inner products are zero. At a given extraction radius $r_{\text {ext }}$ we perform a multipolar decomposition by projecting $\Psi_{4}$ onto a spherical harmonic basis of spin weight $s=-2$ as

$$
\Psi_{4}(t, r, \theta, \varphi)=\sum_{\ell, m} \psi_{4}^{\ell, m}(t, r)_{-2} Y_{\ell, m}(\theta, \varphi)
$$

whose relation with the second time derivative of the two polarizations of the GW strain is given by

$$
\psi_{4}^{\ell, m}(t, r)=\ddot{h}_{\ell, m}^{+}-i \ddot{h}_{\ell, m}^{\times} .
$$
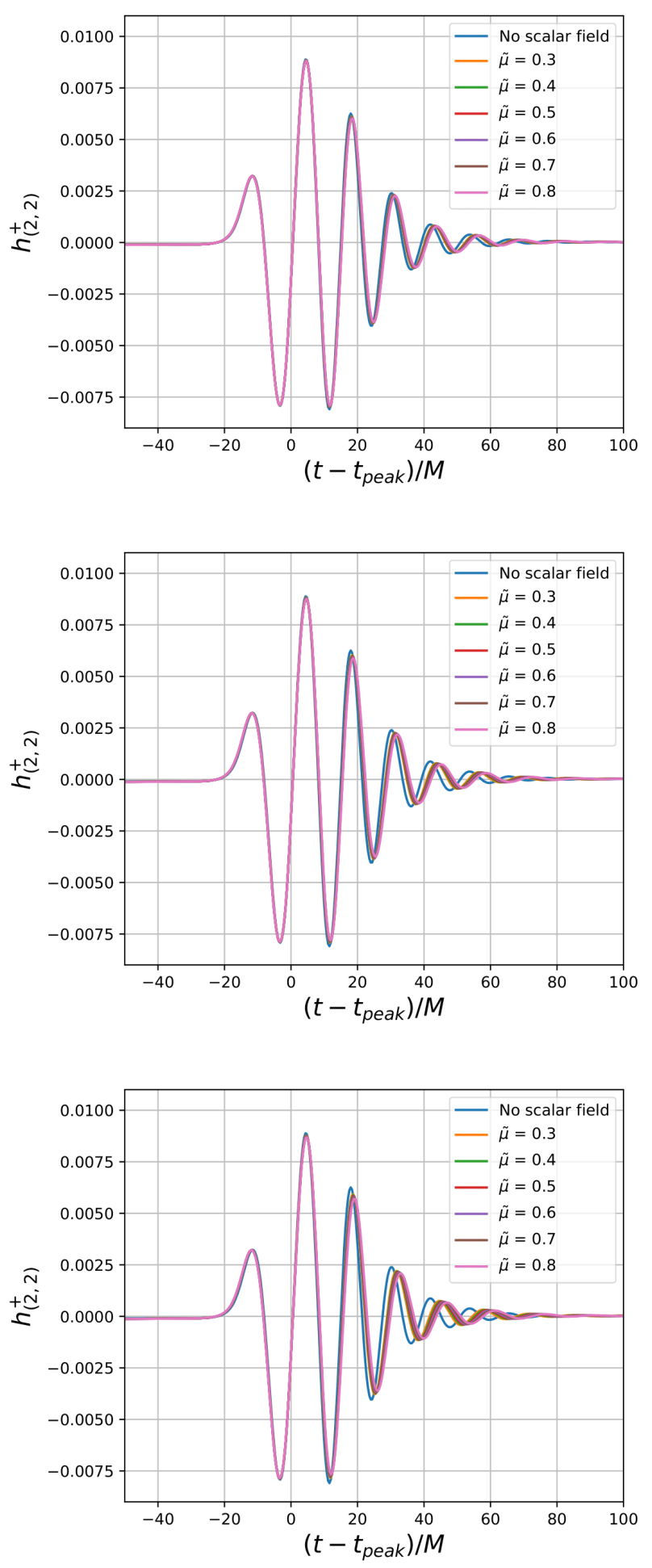

FIG. 1. The $l=m=2$ mode of the real part of the GW strain $h_{+}$extracted at $r_{\text {ext }}=40 M$. Different curves correspond to varying values of the scalar field mass parameter $\tilde{\mu}$ for the field amplitude $\phi_{0}=3.5 \times 10^{-3}$ (top panel), $\phi_{0}=4.5 \times 10^{-3}$ (middle panel), and $\phi_{0}=5.5 \times 10^{-3}$ (bottom panel). 


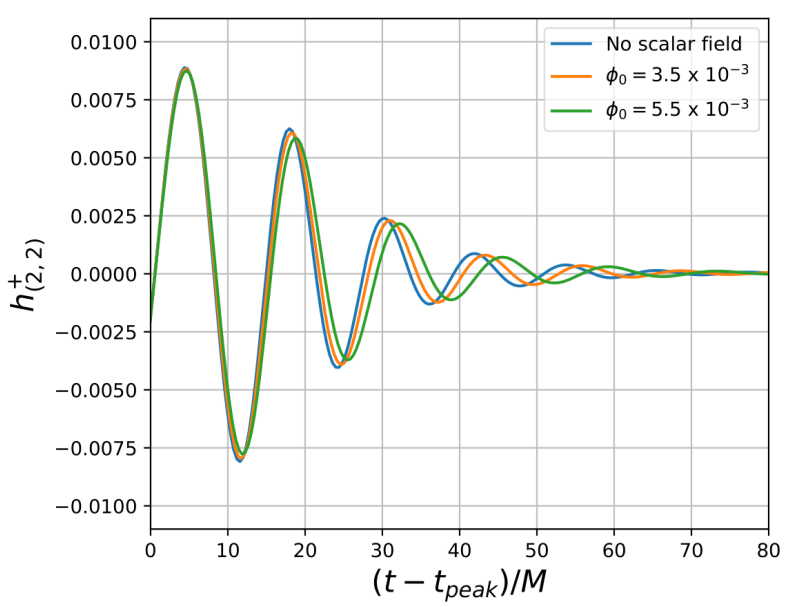

FIG. 2. Comparison of the ringdown quadrupolar GW strains of BBH mergers with and without scalar field content. The former corresponds to the $\tilde{\mu}=0.5$ case with $\phi_{0}=3.5 \times 10^{-3}$ and $5.5 \times 10^{-3}$. The labels here are the same as in Fig. 1 .

We use the post-processing python package PYGWAnALYSIS [89] to convert $\Psi_{4}$ data to GW strain. Figure 1 displays the real part of the dominant quadrupolar $(l=m=2)$ mode of the GW strain $\left(h^{+}\right)$for varying values of the mass of the scalar field and of its initial amplitude. The signal is extracted at $r_{\text {ext }}=40 M$ and $t_{\text {peak }}$ refers to the instant of time when the norm of the strain waveform, $\left|h_{2,2}\right|$, reaches its maximum. This figure shows that the presence of the scalar field produces a shift in the signal compared to the vacuum BBH case. This shift is most visible in the ringdown part of the signal and becomes larger the larger the values of $\tilde{\mu}$ and $\phi_{0}$.

The stronger and faster damping observed during the ringdown in the presence of high-amplitude scalar fields is highlighted in Figure 2. This figure compares the waveforms for $\phi_{0}=3.5 \times 10^{-3}$ and $5.5 \times 10^{-3}$, both for $\tilde{\mu}=0.5$, with a $\mathrm{BBH}$ merger with no scalar field content. In order to quantify this effect and to study the distinguishability of $\phi_{0}$ in actual GW observations, we carry out Bayesian inference with our waveform models. This is discussed in the next section.

\section{MEASURING SCALAR FIELD PARAMETERS IN OBSERVATIONS OF BBH MERGERS}

With multiple BBH merger detections in the past and several tens to hundred expected in ground-based detectors in the coming years, it will likely become possible to distinguish BBHs with sufficiently large scalar field amplitudes from those without any such field - or at least constrain the presence of such fields in BBH mergers. To estimate how precisely one will be able to do so, we fitted several models to the post-merger parts of our numerical-relativity waveforms, out of which the chirp sine-Gaussian waveform model came out to be the most suitable one - partly motivated by the exponentially damped sinusoid nature of the signal in the absence of a scalar field (see, e.g., [90, 91] and references therein). This is due to both its simplicity in structure and the small number of parameters it employs, as well as to its very high match $(\gtrsim 95 \%)$ with the numerical waveforms. The chirp sine-Gaussian form is described by the GW strain

$$
g\left(t ; f_{0}, Q, \kappa\right) \equiv A e^{-4 \pi^{2} f_{0}^{2} t^{2} / Q^{2}} \cos \left(2 \pi f_{0} t+\kappa t^{2}\right),
$$

where $Q$ is the quality factor that dictates the damping time, $f_{0}$ the central frequency of the sinusoid, and $\kappa$ the "chirp parameter" quantifying the rate of change of frequency with time (see [92] for details).

\section{A. Bayesian parameter estimation}

To assess how well the analytical model of our ringdown signals in Eq. (9) matches the numerical waveforms, and the precision as well as accuracy with which one can measure the $\mathrm{BBH}$ and scalar field parameters, we use Bayesian inference. The code employed to implement it on our simulated detector data and signals is BILBY [93], which is primarily designed for inferring the parameters of compact binary coalescence signals. It provides both Nested sampler and Markov Chain Monte Carlo (MCMC) sampler options for computing the parameter posteriors of modeled waveforms. In our study we used the nested sampler "Dynesty" $[94,95]$ since it is a more appropriate choice when only a few parameters characterize a large set of waveforms.

To perform this Bayesian analysis, the numerical waveforms are scaled such that for each set of parameter error estimates, the source is always kept at a fixed distance, namely at $450 \mathrm{Mpc}$. Increasing the source distance leads to an increase in parameter errors that can make it difficult to distinguish the scalar-field values simulated here.

We use the IMRPhENOMD waveform templates [96, 97] with lower-frequency cut-off set high enough in our analysis so as to utilize only the post-merger parts of the signals for computing the signal-to-noise ratios (SNRs) and the parameter estimates. In particular, parameter estimation is performed using the quadrupolar $(l=m=$ 2) mode of the GW strain waveform as described in Section IIIB. We truncate every waveform such that only the cycles following the peak amplitude are retained. All waveforms are injected in simulated zero-mean, colored Gaussian noise using the aLIGO's zero-detuned-highpower noise power spectral density (PSD) [98]. The timeaxis of the numerical waveform was scaled by setting up the component masses such that the signal frequency lies in the aLIGO sensitivity band. The component masses that we need for this purpose are of the order of $40 M_{\odot}$ and the mass of the scalar particle is in the range be- 

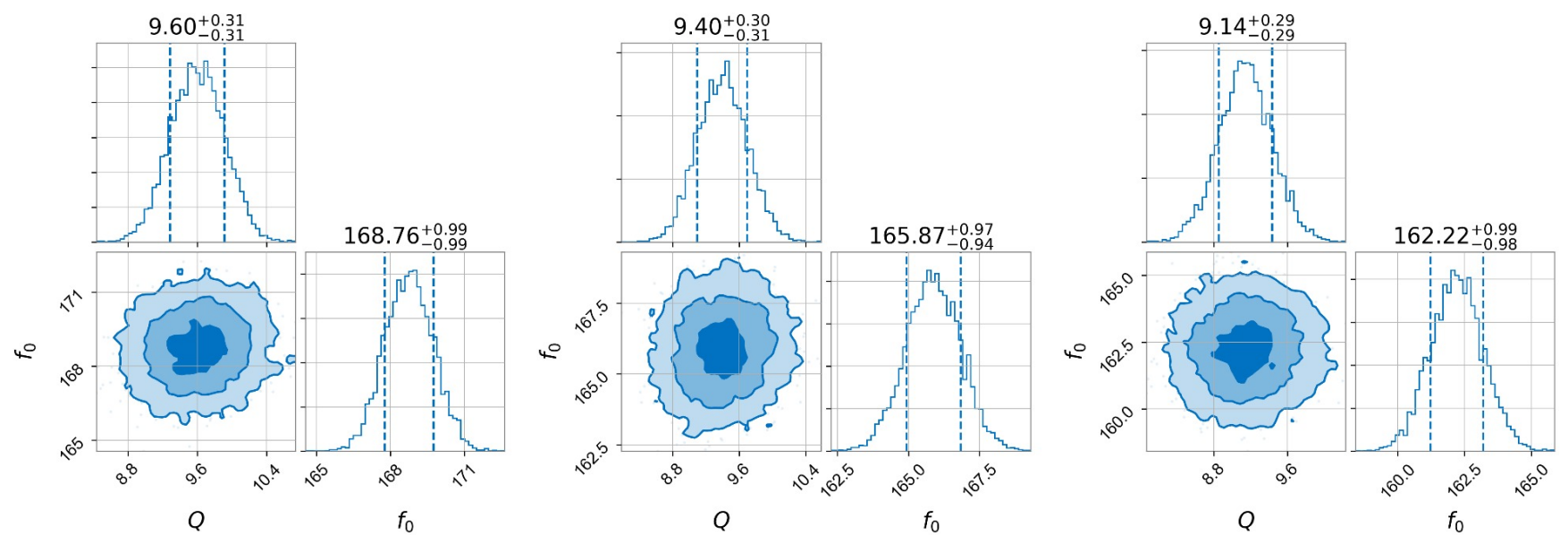

FIG. 3. Corner plots showing the posterior distributions of $f_{0}$ and $Q$ of our chirp sine-Gaussian waveform model for $\phi_{0}=$ $3.5 \times 10^{-3}$ (left), $\phi_{0}=4.5 \times 10^{-3}$ (middle), and $\phi_{0}=5.5 \times 10^{-3}$ (right). All three cases correspond to $\tilde{\mu}=0.50$.

\begin{tabular}{|l|l|l|}
\hline \multicolumn{3}{|c|}{ Best Parameter fits for 100 Mpc } \\
\hline$\phi_{0}$ and $\tilde{\mu}$ & $\mathrm{Q}$ & $f_{0}(\mathrm{~Hz})$ \\
\hline $0 ; 0$ (no scalar field) & $9.89_{-32}^{+34}$ & $173.54_{-0.94}^{+0.98}$ \\
\hline Case $\phi_{0}=3.5 \times 10^{-3}$ & \multicolumn{3}{|c|}{} \\
\hline 0.30 & $9.66_{-0.32}^{+0.32}$ & $169.76_{-0.99}^{+0.96}$ \\
0.40 & $9.62_{-0.31}^{+0.31}$ & $169.25_{-0.94}^{+0.93}$ \\
0.50 & $9.60_{-0.31}^{+0.31}$ & $168.76_{-0.99}^{+0.99}$ \\
0.60 & $9.56_{-0.30}^{+0.30}$ & $168.28_{-0.95}^{+0.96}$ \\
0.70 & $9.53_{-0.31}^{+0.30}$ & $167.83_{-1.00}^{+0.94}$ \\
0.80 & $9.51_{-0.32}^{+0.30}$ & $167.21_{-1.01}^{+0.96}$ \\
\hline Case $\phi_{0}=4.5 \times 10^{-3}$ & \multicolumn{3}{|c|}{} \\
\hline 0.30 & $9.51_{-0.30}^{+0.31}$ & $167.39_{-0.95}^{+0.94}$ \\
0.40 & $9.47_{-0.31}^{+0.31}$ & $166.52_{-0.92}^{+0.95}$ \\
0.50 & $9.40_{-0.30}^{+0.30}$ & $165.87_{-0.94}^{+0.97}$ \\
0.60 & $9.34_{-0.30}^{+0.31}$ & $165.06_{-0.96}^{+0.95}$ \\
0.70 & $9.28_{-0.29}^{+0.31}$ & $164.24_{-0.96}^{+0.99}$ \\
0.80 & $9.22_{-0.29}^{+0.28}$ & $163.35_{-0.98}^{+0.93}$ \\
\hline Case $\phi_{0}=5.5 \times 10^{-3}$ & \multicolumn{3}{|c|}{} \\
\hline 0.30 & $9.32_{-0.31}^{+0.29}$ & $164.43_{-0.96}^{+0.92}$ \\
0.40 & $9.23_{-0.30}^{+0.28}$ & $163.26_{-1.02}^{+0.96}$ \\
0.50 & $9.14_{-0.29}^{+0.29}$ & $162.22_{-0.98}^{+0.99}$ \\
0.60 & $9.06_{-0.29}^{+0.28}$ & $161.16_{-0.99}^{+0.96}$ \\
0.70 & $8.99_{-0.30}^{+0.28}$ & $159.87_{-0.94}^{+0.97}$ \\
0.80 & $8.89_{-0.28}^{+0.28}$ & $158.48_{-0.95}^{+0.96}$ \\
\hline
\end{tabular}

TABLE I. Variation in medians and $90 \%$ errors in $Q$ and $f_{0}$ with $\phi_{0}$ and $\tilde{\mu}$. Here $\tilde{\mu}$ varies from 0.3 to 0.8 for three values of the scalar field: $\phi_{0}=3.5 \times 10^{-3}, 4.5 \times 10^{-3}$ and $5.5 \times 10^{-3}$. The error bars are for a source distance of $100 \mathrm{Mpc}$. These variations are plotted in figures 6 (left panel) and 7 .

tween $0.5 \times 10^{-12} \mathrm{eV}$ and $1.4 \times 10^{-12} \mathrm{eV}$. Further details about the procedure are provided in Appendix B.

The priors used for the parameters $Q$ and $f_{0}$ are uniform, with $Q \in[6,14]$ and $f_{0} \in[140,190] \mathrm{Hz}$ and the likelihood used is Gaussian with sigma set equal to the standard deviation of aLIGO noise. The posteriors thus calculated give us the estimated values and the $1 \sigma$ error

\begin{tabular}{|l|l|}
\hline \multicolumn{2}{|c|}{ Best Parameter fits for $450 \mathrm{Mpc}$} \\
\hline$\phi_{0}$ and $\tilde{\mu}$ & $f_{0}(\mathrm{~Hz})$ \\
\hline $0 ; 0 \quad$ (no scalar field) & $174.00_{-4.34}^{+4.19}$ \\
\hline Case $\phi_{0}=4.5 \times 10^{-3}$ & \\
\hline 0.30 & $168.10_{-4.22}^{+4.44}$ \\
0.40 & $167.29_{-4.41}^{+4.45}$ \\
0.50 & $166.67_{-4.45}^{+4.60}$ \\
0.60 & $165.81_{-4.38}^{+4.49}$ \\
0.70 & $165.00_{-4.39}^{+4.52}$ \\
0.80 & $164.10_{-4.50}^{+4.70}$ \\
\hline Case $\phi_{0}=5.5 \times 10^{-3}$ & \\
\hline 0.30 & $165.29_{-4.31}^{+4.39}$ \\
0.40 & $164.08_{-4.52}^{+4.56}$ \\
0.50 & $163.14_{-4.48}^{+4.45}$ \\
0.60 & $162.14_{-4.61}^{+4.60}$ \\
0.70 & $160.95_{-4.58}^{+4.54}$ \\
0.80 & $159.65_{-4.72}^{+4.65}$ \\
\hline
\end{tabular}

TABLE II. Variation in medians and $90 \%$ errors in $f_{0}$ with $\phi_{0}$ and $\tilde{\mu}$ for a source distance of $450 \mathrm{Mpc}$. These variations are plotted in the right panel of Fig. 6. The values for parameter $Q$ are not included in the table as their error bars overlap with the no-scalar-field case.

bars. Some illustrative corner plots of those posteriors are shown in Fig. 3. As mentioned before, we consider three scalar-field cases with $\tilde{\mu}$ ranging from 0.3 to 0.8 . In physical units this corresponds to $0.7 \times 10^{-12} \mathrm{eV}$ $1.8 \times 10^{-12} \mathrm{eV}$ for BBH with each component of the order of $30 M_{\odot}$ and to $3.4 \times 10^{-13} \mathrm{eV}-9 \times 10^{-13} \mathrm{eV}$ for $\mathrm{BBH}$ with $60 M_{\odot}$ components.

The $\kappa$ parameter is fixed to $13000 \mathrm{~Hz}^{2}$ in all cases as we observed it does not vary much for different values of $\phi_{0}$ and $\tilde{\mu}$. It only shows a variation of $15 \%$ when set free but does not have much effect on the match (which changes by $<1 \%$ ). Lists of estimated parameters are shown in Tables I and II for source distances of $100 \mathrm{Mpc}$ and $450 \mathrm{Mpc}$, respectively. 


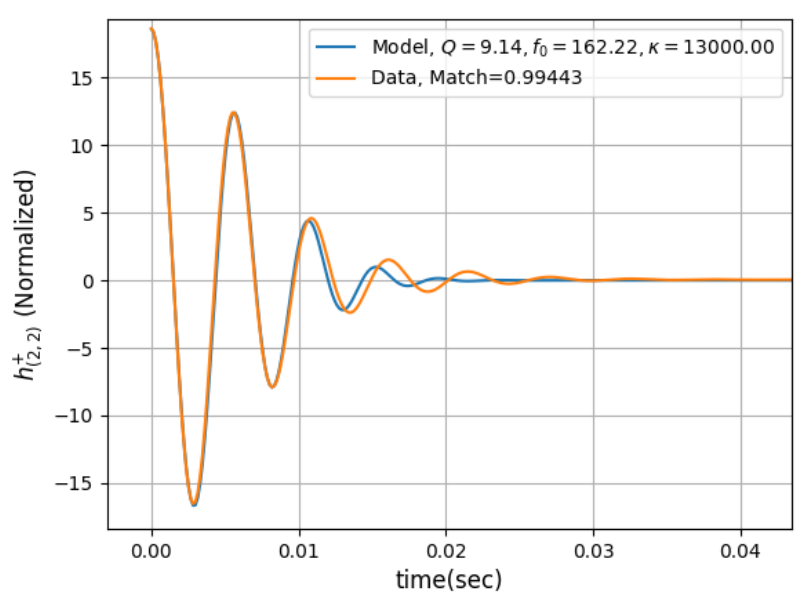

FIG. 4. Comparison of the post-merger waveform against the chirp sine-Gaussian fitted model for the case $\phi_{0}=5.5 \times 10^{-3}$ and $\tilde{\mu}=0.5$. It yields more than $99 \%$ match.

By studying injections at very high SNRs we confirmed that the systematic error in the estimated parameters, especially, $f_{0}$ as listed in Table I (where the source distance is taken at $100 \mathrm{Mpc}$ ), is no more than $\sim 0.1 \%$. With these estimated parameters our model gives more than 99\% match with the post merger part of the waveform, as shown in Fig. 4 for the particular case $\phi_{0}=5.5 \times 10^{-3}$ and $\tilde{\mu}=0.5$.

To validate our parameter estimation method we performed some tests by checking how accurate it estimated the parameters $f_{0}, Q$ and $\kappa$ of simulated chirping sineGaussian signals. For this purpose, we varied $\kappa$ from 12000 to $14700 \mathrm{~Hz}^{2}$ in steps of $300 \mathrm{~Hz}^{2}$. We did it for two cases. In the first case we fixed $f_{0}=157.0 \mathrm{~Hz}, Q=9.2$ and in the second case $f_{0}=145.0 \mathrm{~Hz}, Q=8.6$. The results, shown in Fig. 5, demonstrate the effectiveness of our method in recovering the signal parameters.

\section{B. Revealing scalar fields properties by measuring $f_{0}$}

The signal parameter $f_{0}$ is an accurate tracker of the strength of the scalar field, $\phi_{0}$, so long as we allow for its variation with the parameter $\tilde{\mu}$. It is worth mentioning that for a fixed value of $\tilde{\mu}$, as the value of $\phi_{0}$ increases the mass of the scalar field cloud grows. We use the chirping sine-Gaussian templates in BILBY, as given by equation (9), to measure the values of $f_{0}$, along with $Q$, for the multiple numerical-relativity waveforms simulated for various scalar field configurations. Specifically, we performed parameter estimation for our three values of $\phi_{0}$, namely, $3.5 \times 10^{-3}, 4.5 \times 10^{-3}$, and $5.5 \times 10^{-3}$, as well as for our six values of $\tilde{\mu}$ ranging from 0.3 to 0.8 . This range of values of $\phi_{0}$ allow us to study scalar clouds having less than 10-15\% of the mass of the binary.
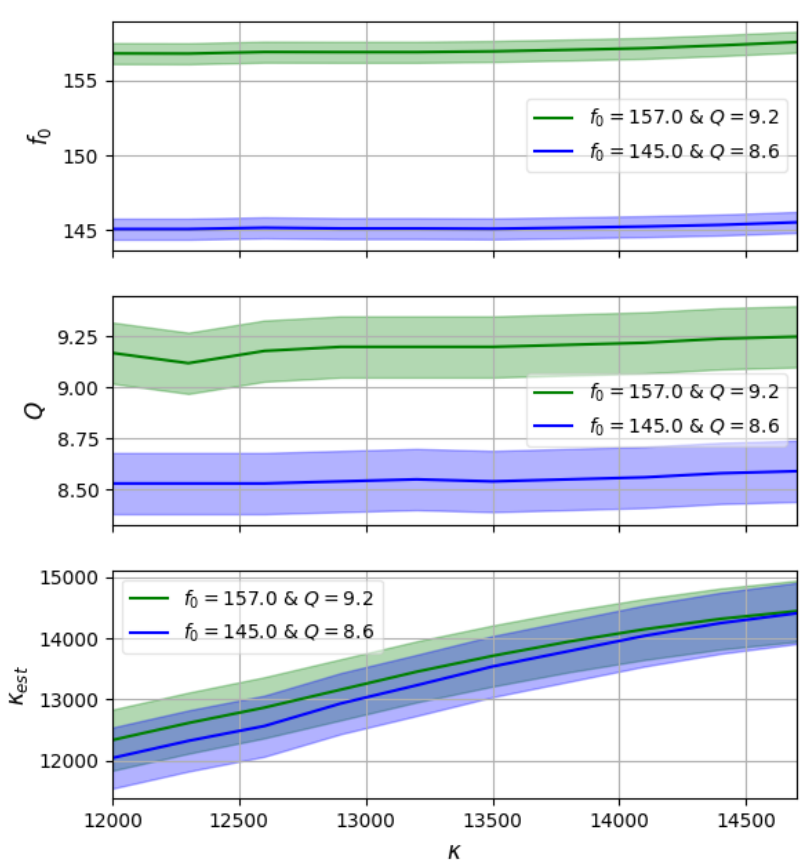

FIG. 5. Results of our validation study for $f_{0}$ (top), $Q$ (middle), and $\kappa_{\text {est }}$ (bottom) for varying values of $\kappa$.

Figures 6 and 7 show the variation of model parameters $f_{0}$ and $Q$, respectively, with the numerical waveform parameter $\tilde{\mu}$. The closer the source the better the results. If the source is at $100 \mathrm{Mpc}$ (left panel of Fig. 6 and Fig. 7) for which the match-filtering SNR value using the post-merger part of template is $\approx 210$, the error bars in the measurements of $Q$ are overlapping for our choices of scalar field strengths. However, the error bars in $f_{0}$ are separate and all of the 18 cases considered can be distinguished from one another, and from the no scalar-field case (with error and median values shown in Table I).

We note that even if the source is at a distance of 200 $\mathrm{Mpc}$, one finds that the error-bars for $f_{0}$ remain separable for much of the $\tilde{\mu}$ range studied here. However, at larger source distances the situation worsens and it is only possible to distinguish stronger scalar fields from the no-scalar-field BBH mergers. This is shown in the right panel of Fig. 6 for a source located at $450 \mathrm{Mpc}$ for which match-filtering SNR value using post-merger part of template is $\approx 45$, a distance around or above the values at which most GW signals from BBH coalescence events are observed by the Advanced LIGO and Advanced Virgo detector network.

With the previous results we can attempt to fit $f_{0}$ as a function of $\tilde{\mu}$

$$
f_{0}(\tilde{\mu})=a \tilde{\mu}+b .
$$

The fits corresponding to the different values of $\phi_{0}$ for 

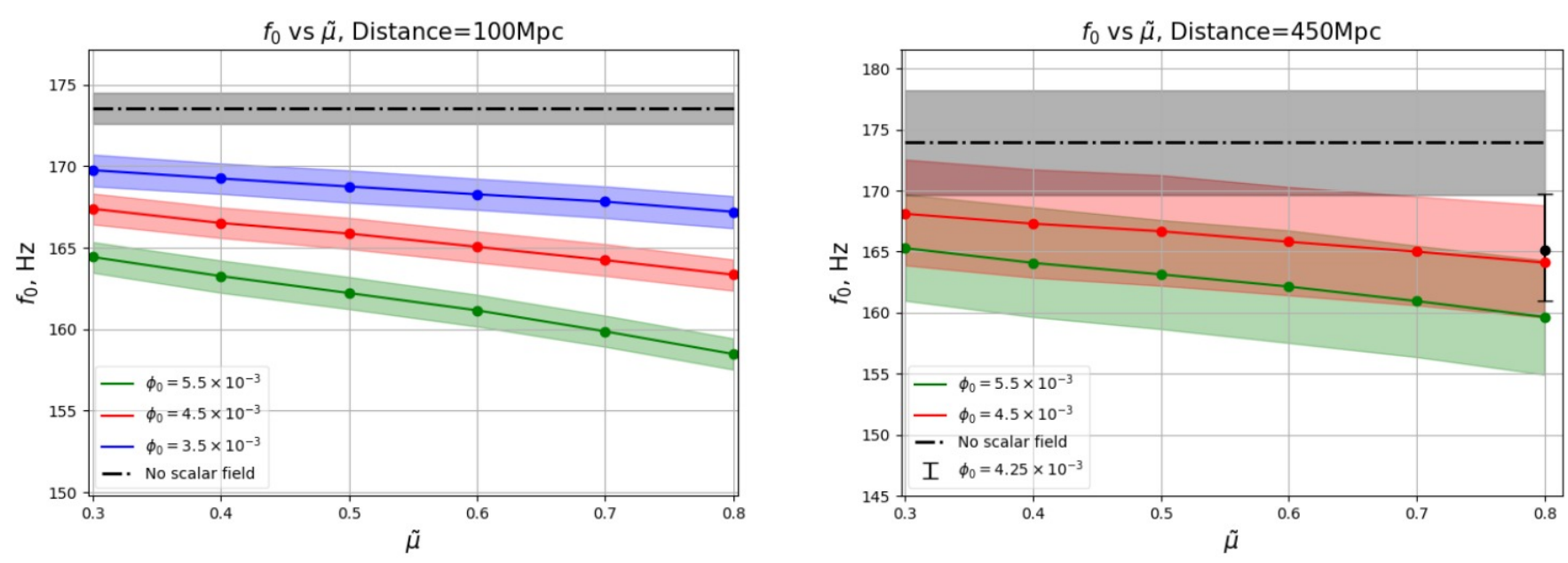

FIG. 6. (Left Panel) The medians and $1 \sigma$ error regions (with interpolations) of the $f_{0}$ posteriors plotted as functions of $\tilde{\mu}$ for the three values of $\phi_{0}$ and for a source located at a distance of $100 \mathrm{Mpc}$. The filled circles denote the values of $\tilde{\mu}$ where the posteriors were individually computed. The value of $f_{0}$ for the no-scalar field case is $173.54_{-0.94}^{+0.98} \mathrm{~Hz}$ and it is shown as a horizontal line in the plot for reference. (Right Panel) Plot of $f_{0}$ vs $\tilde{\mu}$ for source distance of 450 Mpc. The frequency estimate for the no-scalar field case, $f_{0}=174.0_{4.34}^{4.19}$, cannot be distinguished from the estimate for the $\phi_{0}=3.5 \times 10^{-3}$ case for $\tilde{\mu}<0.7$; it can, however, be distinguished from the $\phi_{0}=4.5 \times 10^{-3}$ case for all $\tilde{\mu}$ except close to 0.3 . The figure also shows that the $\phi_{0}=4.25 \times 10^{-3}$ case is the limiting value of $\phi_{0}$ that can be distinguished from the no-scalar field case, close to $\tilde{\mu}=0.8$

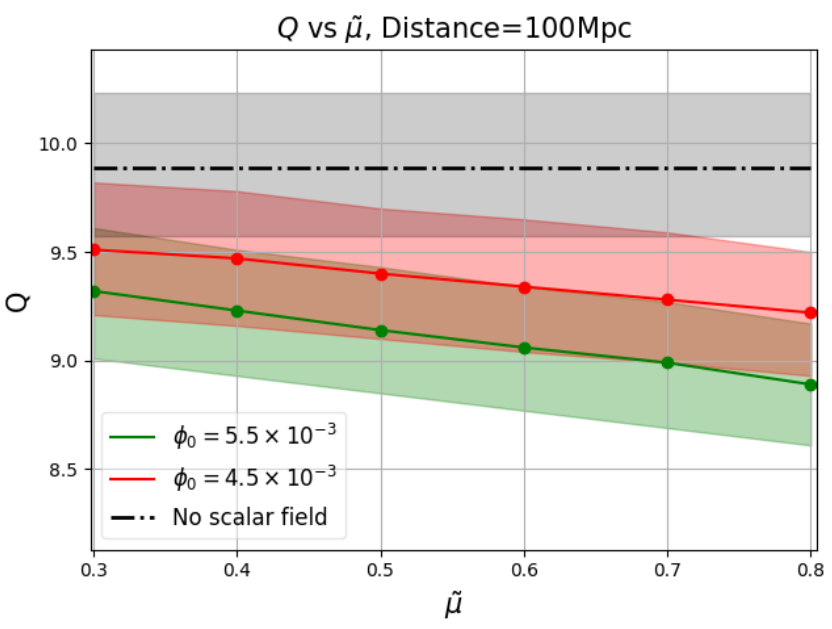

FIG. 7. Median and $1 \sigma$ error regions of the $Q$ posteriors as a function of $\tilde{\mu}$ for $\phi_{0}=5.5 \times 10^{-3}$ and $\phi_{0}=4.5 \times 10^{-3}$ and for a source at a distance of $100 \mathrm{Mpc}$. The value of $Q$ for the no scalar field case is $9.89_{-32}^{+34}$.

the source at a distance of $100 \mathrm{Mpc}$ are plotted in Fig. 8. Values of the coefficients $a$ and $b$ are listed in Table III. As can be seen from the fits (Table III) and figures 6 and 8 , the frequency $f_{0}$ shows a clear dependence on $\phi_{0}$ and $\tilde{\mu}$ and its measurement can be used to put bounds on the characteristics of the source.

To quantify it further we plot in Fig. 9 isocontours of $f_{0}\left(\tilde{\mu}, \phi_{0}\right)$. An isocontour of $f_{0}$ specifies the region of the parameter space that is allowed by the measured value

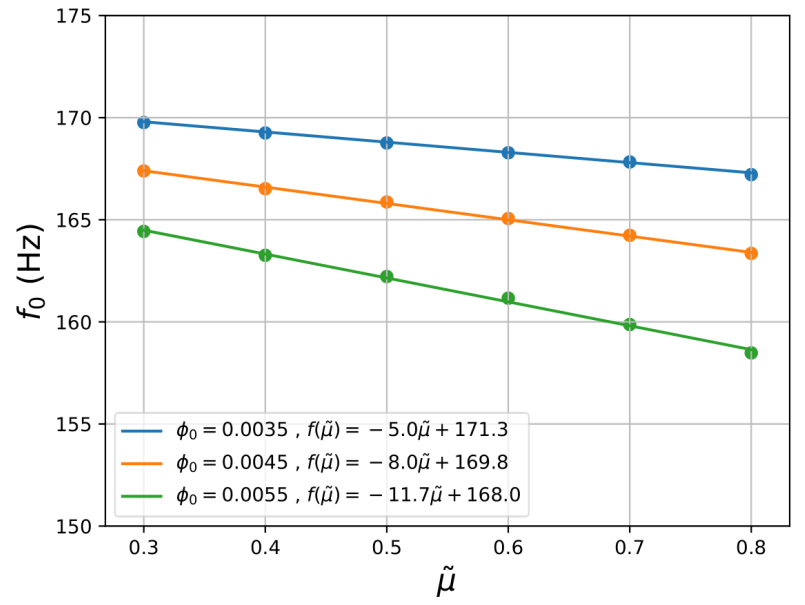

FIG. 8. Linear fits for $f_{0}$ as a function of $\tilde{\mu}, f_{0}(\tilde{\mu})=a \tilde{\mu}+b$, for our three values of $\phi_{0}$.

of $f_{0}$. For example, if an observed GW signal has a frequency $f_{0}=166 \mathrm{~Hz}$, then this implies that in the range $\tilde{\mu} \in[0.3,0.8], \phi_{0}$ must lie between $\sim 3.8 \times 10^{-3}$ and $\sim 5.0 \times 10^{-3}$. Since the measured $f_{0}$ value will typically lie in a confidence interval, the range of $\phi_{0}$ too will have a corresponding spread. Furthermore, if the value of $Q$ can be measured as well with some precision, then along with $f_{0}$ it will provide a measurement of the important quantity $\tilde{\mu}$ characterizing the scalar field cloud. As suggested by Fig. 7 this quest might be elusive unless we detect a golden binary with a large SNR. 


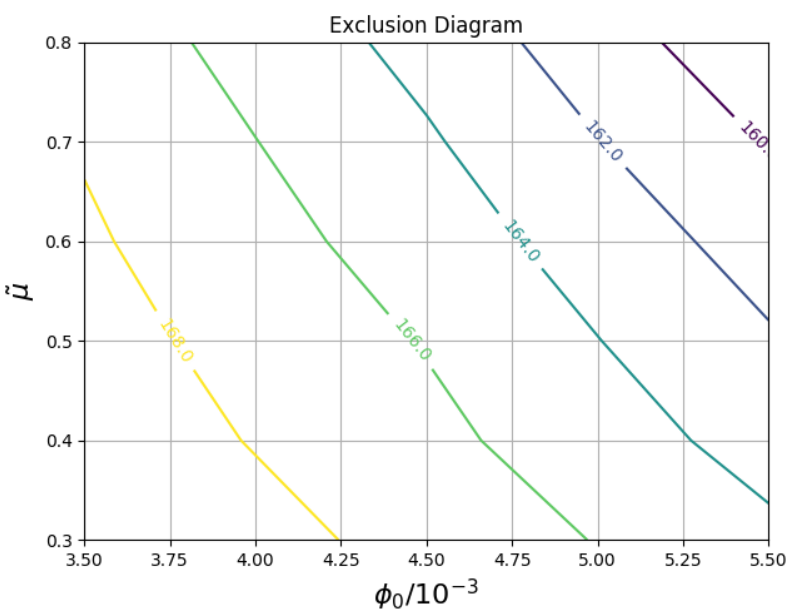

FIG. 9. Isocontours of $f_{0}$ in the $\phi_{0}-\tilde{\mu}$ plane for a source at $100 \mathrm{Mpc}$.

\begin{tabular}{|l|l|l|}
\hline \multicolumn{3}{|c|}{ Model Coefficients } \\
\hline$\phi_{0}$ & $a(\mathrm{~Hz})$ & $b(\mathrm{~Hz})$ \\
\hline 0.0035 & -5.0 & 171.3 \\
0.0045 & -8.0 & 169.8 \\
0.0055 & -11.7 & 168.0 \\
\hline
\end{tabular}

TABLE III. Coefficients of fitting model for $f_{0}$, described by Eqs. (10) for the three values of the scalar field, $\phi_{0}=3.5 \times$ $10^{-3}, 4.5 \times 10^{-3}$ and $5.5 \times 10^{-3}$ at $100 \mathrm{Mpc}$.

\section{CONCLUSIONS}

Massive scalar fields surrounding stationary and nonrotating BHs can form long-lived, quasi-bound states, or clouds, as a result of the presence of a potential well due to the mass term [62]. For rotating BHs, the superradiant instability [42] leads to the formation of hairy BHs Kerr BHs surrounded by bosonic (scalar or vector) hair in which the frequency of the field is synchronized with the angular velocity of the $\mathrm{BH}[47,48]$. Using numerical reativity simulations we have studied mergers of $\mathrm{BBH}$ systems dressed in such scalar field clouds. Our aim has been to find out whether GW observations of BBH mergers could constrain the physical characteristics of a scalar field cloud surrounding those compact binaries. We have considered equal mass BBH systems endowed with Gaussian distributions of scalar field clouds parameterized by their mass $\tilde{\mu}$ and strength $\phi_{0}$, analyzing the imprints on the GWs generated during the mergers. We have numerically simulated the last three quarters of the final orbit prior to merger for a large set of initial models, restricting our analysis to the post-merger phase.

The waveforms extracted from our simulations have revealed that larger values of $\tilde{\mu}$ or $\phi_{0}$ cause bigger changes in the amplitude and frequency of the ringdown part of the signals. The ringdown signals of our mergers can be simulated analytically as chirping sine-Gaussians, char- acterized by only three parameters, returning match values with our numerical-relativity waveforms in excess of $95 \%$. This is not surprising since BBH ringdown signals in General Relativity are damped sinusoids that can be modelled with only two parameters [99]. Using our chirping sine-Gaussian signal model we have carried out computationally expensive Bayesian studies for estimating the parameters of BBH binaries endowed with scalar field clouds. We have been able to establish that the central frequency of the model, $f_{0}$, has a strong dependence on the scalar-field strength $\phi_{0}$ and a weak dependence on $\tilde{\mu}$. Therefore, at a fixed value of $\tilde{\mu}$, a measurement of the signal parameter $f_{0}$ leads to a measurement of $\phi_{0}$. In particular, we have shown that it is possible to distinguish from observations of $\mathrm{BBH}$ mergers at distances of $450 \mathrm{Mpc}$, BBHs without any scalar field from those with a field strength $\phi_{0}=5.5 \times 10^{-3}$, at any fixed value of $\tilde{\mu} \in[0.3,0.8]$, with $90 \%$ confidence, or better. We have shown that aLIGO may have the potential to distinguish between the GW signal produced by a BBH system with components of $40 M_{\odot}$ if the binary is immersed in a cloud of boson particles with masses between $5 \times 10^{-13} \mathrm{eV}$ and $1.4 \times 10^{-12} \mathrm{eV}$ from the $\mathrm{BBH}$ with the same range of masses in vacuum.

We take these results as encouraging indications for the prospect of constraining scalar field clouds in $\mathrm{BBH}$ observations. However, to assess their utility for real observations, one must study the impact of a wider parameter space of the binaries on how accurately and precisely one will be able to measure the scalar field parameters from the waveforms, while also battling possible parameter degeneracies that can arise. For instance, the same $f_{0}$ and $Q$ values can correspond to a variety of $\mathrm{BBH}$ remnant masses and spins as well as scalar-field parameters. (Arguably, some of these degeneracies may break or get mitigated by measurements of source parameters in the inspiral part of the signals.) To address this issue, one will need to simulate waveforms for a broader range of astrophysically relevant $\mathrm{BBH}$ component masses and spins, and scalar-field parameters, and analyze the parameter degeneracies that might arise as well as their possible resolution. We leave this computationally expensive study for the future.

While in this investigation we have limited ourselves to single BBH observations, we note that by combining multiple $\mathrm{BBH}$ detections, one may be able to constrain the scalar field configurations in these mergers collectively for populations. Such an exercise will be similar to stacking ringdown signals from multiple $\mathrm{BBH}$ signals to, e.g., test the no-hair theorem. In our case, however, a straightforward extension to populations is complicated by the possibility that the scalar field parameters may vary from one BBH source to another. Similarly, it would be interesting to explore if BBH observations can be used to determine or constrain the mass of ultra-light bosons. We plan to pursue these prospects in a future work. 


\section{ACKNOWLEDGMENTS}

We are thankful to Cristiano Palomba and the referee for their useful comments and suggestions. This research is supported by the Spanish Agencia Estatal de Investigación (grants PGC2018-095984-B-I00), by the Generalitat Valenciana (PROMETEO/2019/071), by the European Union's Horizon 2020 RISE programme H2020MSCA-RISE-2017 (Grant No. FunFiCO-777740) and by DGAPA-UNAM through grant IN105920. A. G. is supported, in part, by the Navajbai Ratan Tata Trust research grant. NSG is supported by the Fundação para a Ciência e a Tecnologia (FCT) projects PTDC/FISOUT/28407/2017, UID/FIS/00099/2020 (CENTRA), and CERN/FIS-PAR/0027/2019. The numerical simulations for this work were performed on the Perseus and Pegasus clusters, which are part of the high performance computing (HPC) facility at The Inter-University Centre for Astronomy and Astrophysics, Pune, India (IUCAA). We would also like to acknowledge help and support of their staff.

\section{Appendix A: Constraint violation in the presence of a scalar field}

As described in section III A, the introduction of a scalar-field distribution in an otherwise constraintpreserving $\mathrm{BBH}$ initial setup leads to constraint violations. To make sure that our numerical simulations are still valid for the time evolution under consideration, we compute the changes in the mass and the area of the black hole and compare those values with the no scalar field scenario.

The top panel of Figure 10 shows the percentage change in mass whereas the bottom panel indicates the corresponding change in area, for the largest mass parameter considered, $\tilde{\mu}=0.8$, and for three values of the field strength.

To compute the area, mass and other physical quantities on the horizon, first we find the horizon using the AHFinderDiRECT thorn $[74,75]$ followed by the application of the QUASILOCALMEASURES thorn, which implements the isolated and dynamical horizon framework [100-102]. This framework enables the computation of quasi-local quantities on marginally trapped surfaces such as the apparent horizon. Recently, the correlation between the shear of the horizon and the News function in the wave-zone was demonstrated for quasi-circular BBH mergers $[103,104]$ and horizon dynamics was studied in terms of the shear and the multipole moments for the head-on collision of two black holes [105]. These works could be important steps towards inferring finer details of black hole horizon properties through gravitational-wave observations. It might be interesting to pursue similar studies in the presence of scalar field clouds, which we aim to do in the future.

In Fig. 11 we show the evolution of the $L_{2}$-norm of the
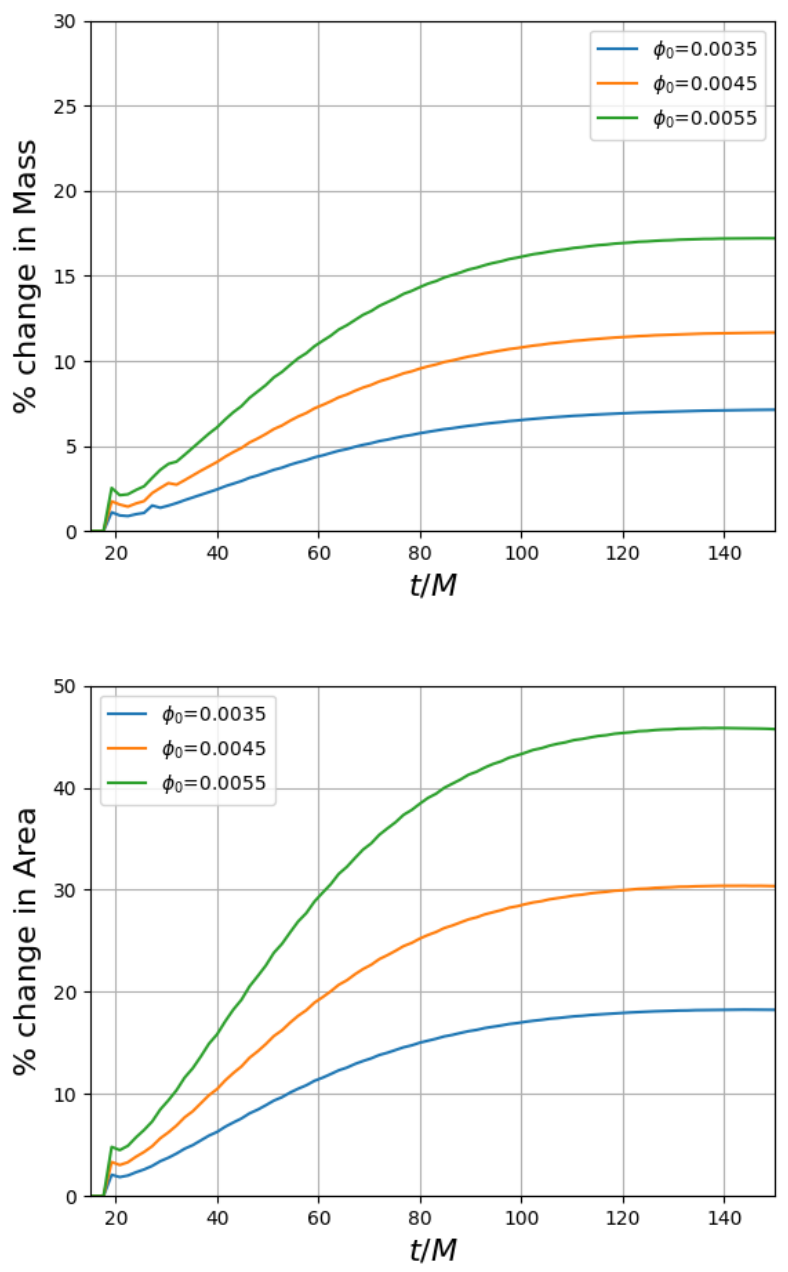

FIG. 10. Percentage change in mass (top panel) and area (bottom panel) with respect to the no scalar field case for the largest mass parameter considered $\tilde{\mu}=0.8$ and for field strength $\phi_{0}=3.5 \times 10^{-3}, 4.5 \times 10^{-3}$ and $5.5 \times 10^{-3}$.

Hamiltonian constraint for the simulations of Fig. 10. We find that the magnitude of the violation of the $L_{2}$-norm of the Hamiltonian constraint is comparable to the no scalar field case for the range of field strengths $\phi_{0}$ and mass parameter $\tilde{\mu}$ considered. We note that since these results correspond to the largest mass parameter of our study, the impact of the constraint violation is in general much lower for the $\phi_{0}-\tilde{\mu}$ parameter space investigated.

\section{Appendix B: Pre-parameter estimation treatment of numerical data}

Since the numerical waveforms are computed here in terms of $\psi_{4}$, as functions of time in code units of the Einstein toolkit [70], it is necessary to convert the $\psi_{4}$ data into GW strain data and convert the code time units into seconds (physical units) so that they are usable 


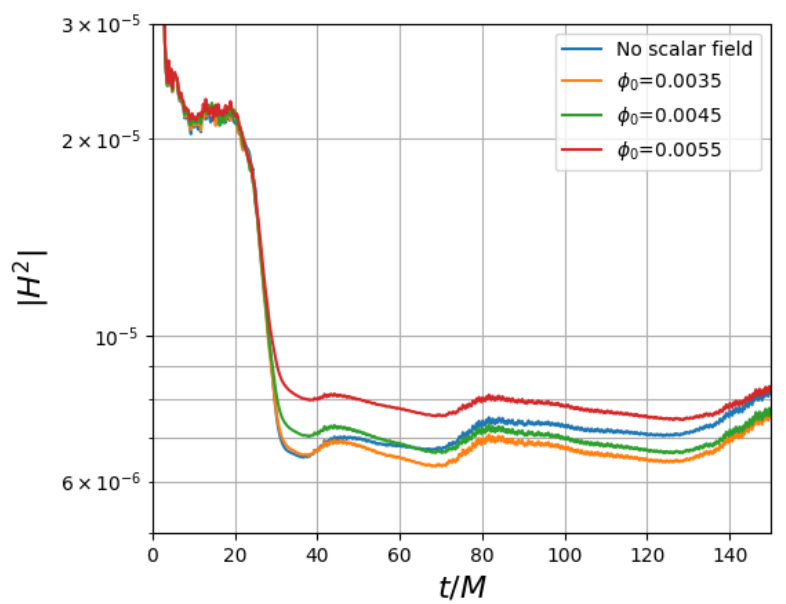

FIG. 11. Time evolution of the $L_{2}$-norm of the Hamiltonian constraint for $\mathrm{BBH}$ mergers with and without a scalar-field cloud. The data shown correspond to the largest mass parameter considered in this work, $\tilde{\mu}=0.8$, for the field strengths $\phi_{0}=3.5 \times 10^{-3}, 4.5 \times 10^{-3}$, and $5.5 \times 10^{-3}$

for GW observations and parameter measurement projections. Here we show how such a conversion is done. The conversion from code units to seconds depends on the total mass of the system $M_{\text {total }}$. This fact can be used to adjust the $\mathrm{BBH}$ mass so as to bring the frequency parameter of the numerical waveforms into aLIGO's most sensitive region band, namely, 100-200 Hz.

Moreover, Some care must be borne when computing the match with numerical relativity (NR) waveforms, as we explain below:

1. The NR simulations produce $\psi_{4}$ data for our various scalar field configurations. Therefore, for GW data analysis, we first construct GW strain waveforms from these data.

2. For labeling the time-points of the strain data in physical units, we used the following conversion

$t($ in sec $)=M_{\text {total }} \times \frac{M_{\odot} G}{c^{3}} *($ cctk_time $)$

where cctk_time refers to the code time units in the Einstein Toolkit [70].

3. Each waveform strain time-series was chopped, resampled and zero padded to prepare it for our analysis with only the post-merger piece (i.e. the part of the NR waveform that starts at the time-point where the peak amplitude is attained in the timedomain); see Fig. 4.

4. As we wanted our waveforms in the aLIGO sensitivity band, we chose the masses such that the frequency parameter falls in that region. To get the approximated value of those masses we calculated the match of numerical waveforms with the IMRPhenomD $[96,97]$ template in the region $(100,200) \mathrm{Hz}$ [the approx aLIGO sensitivity band]. The match comes out to be very high in the mass region of $30-60 M_{\odot}$. On this bases we chose component masses to be $40 M_{\odot}$.

5. We also calculated frequency of the numerical waveforms by measuring the half cycle and concluded that we need around $40-40 M_{\odot} \mathrm{BBH}$ to get our frequency in aLIGO sensitivity band.

6. In our study we analysed numerical waveforms at different source distances. This we achieve by making the peak amplitude of numerical waveforms equal to the PyCBC[106] generated IMRPhenomD template for the distance we want to study.
[1] B. P. Abbott, R. Abbott, T. D. Abbott, S. Abraham, F. Acernese, K. Ackley, C. Adams, R. e. a. Adhikari, LIGO Scientific Collaboration, and Virgo Collaboration, Physical Review X 9, 031040 (2019), arXiv:1811.12907 [astro-ph.HE].

[2] R. Abbott, arXiv e-prints , arXiv:2004.08342 (2020), arXiv:2004.08342 [astro-ph.HE].

[3] B. Abbott et al. (LIGO Scientific, Virgo), Astrophys. J. Lett. 892, L3 (2020), arXiv:2001.01761 [astro-ph.HE].

[4] R. Abbott et al., ApJ 896, L44 (2020).

[5] R. Abbott, others, LIGO Scientific Collaboration, and Virgo Collaboration, Phys. Rev. Lett. 125, 101102 (2020).

[6] B. Abbott et al. (LIGO Scientific, Virgo), Astrophys. J. Lett. 900, L13 (2020).

[7] S. L. Liebling and C. Palenzuela, Living Reviews in Relativity 15, 6 (2012), arXiv:1202.5809 [gr-qc].
[8] L. K. Wong, A.-C. Davis, and R. Gregory, Phys. Rev. D 100, 024010 (2019), arXiv:1903.07080 [hep-th].

[9] N. Guerlebeck and M. Scholtz, (2018), arXiv:1802.05423 [gr-qc].

[10] M. A. S. et al, (2019), arXiv:1908.11375 [gr-qc].

[11] S. Aneesh, S. Bose, and S. Kar, Phys. Rev. D 97, 124004 (2018), arXiv:1803.10204 [gr-qc].

[12] V. Cardoso and P. Pani, Living Reviews in Relativity 22, 4 (2019), arXiv:1904.05363 [gr-qc].

[13] C. Palenzuela, I. Olabarrieta, L. Lehner, and S. L. Liebling, Physical Review D 75, 064005 (2007).

[14] V. Cardoso, S. Hopper, C. F. Macedo, C. Palenzuela, and P. Pani, Physical Review D 94, 084031 (2016).

[15] R. Brito, V. Cardoso, and H. Okawa, Physical review letters 115, 111301 (2015).

[16] R. Brito, V. Cardoso, C. F. Macedo, H. Okawa, and C. Palenzuela, Physical Review D 93, 044045 (2016). 
[17] T. Helfer, E. A. Lim, M. A. Garcia, and M. A. Amin, arXiv preprint arXiv:1802.06733 (2018).

[18] M. Bezares, C. Palenzuela, and C. Bona, Physical Review D 95, 124005 (2017).

[19] C. Palenzuela, P. Pani, M. Bezares, V. Cardoso, L. Lehner, and S. Liebling, Phys. Rev. D 96, 104058 (2017).

[20] T. Dietrich, S. Ossokine, and K. Clough, arXiv preprint arXiv:1807.06959 (2018).

[21] K. Clough, T. Dietrich, and J. C. Niemeyer, Physical Review D 98, 083020 (2018).

[22] M. Bezares and C. Palenzuela, Classical and Quantum Gravity (2018).

[23] N. Sanchis-Gual, C. Herdeiro, J. A. Font, E. Radu, and F. Di Giovanni, Phys. Rev. D 99, 024017 (2019).

[24] L. Barack et al., (2018), arXiv:1806.05195 [gr-qc].

[25] A. Arvanitaki, S. Dimopoulos, S. Dubovsky, N. Kaloper, and J. March-Russell, Phys. Rev. D 81, 123530 (2010), arXiv:0905.4720 [hep-th].

[26] V. Sahni and L.-M. Wang, Phys.Rev. D62, 103517 (2000), arXiv:astro-ph/9910097 [astro-ph].

[27] W. Hu, R. Barkana, and A. Gruzinov, Phys. Rev. Lett. 85, 1158 (2000), arXiv:astro-ph/0003365.

[28] T. Matos and L. A. Urena-Lopez, Class. Quant. Grav. 17, L75 (2000), arXiv:astro-ph/0004332.

[29] T. Matos and L. A. Urena-Lopez, Phys.Rev. D63, 063506 (2001), arXiv:astro-ph/0006024 [astro-ph].

[30] L. Hui, J. P. Ostriker, S. Tremaine, and E. Witten, Phys. Rev. D 95, 043541 (2017), arXiv:1610.08297 [astro-ph.CO].

[31] H. Witek, V. Cardoso, A. Ishibashi, and U. Sperhake, Phys.Rev. D87, 043513 (2013), arXiv:1212.0551 [gr-qc].

[32] J. Barranco, A. Bernal, J. C. Degollado, A. DiezTejedor, M. Megevand, et al., Phys.Rev.Lett. 109, 081102 (2012), arXiv:1207.2153 [gr-qc].

[33] J. Barranco, A. Bernal, J. C. Degollado, A. DiezTejedor, M. Megevand, et al., Phys.Rev. D89, 083006 (2014), arXiv:1312.5808 [gr-qc].

[34] S. R. Dolan, (2012), arXiv:1212.1477 [gr-qc].

[35] A. Cruz-Osorio, F. S. Guzman, and F. D. LoraClavijo, JCAP 1106, 029 (2011), arXiv:1008.0027 [astro-ph.CO].

[36] N. Sanchis-Gual, J. C. Degollado, P. J. Montero, and J. A. Font, Phys. Rev. D 91, 043005 (2015).

[37] N. Sanchis-Gual, J. C. Degollado, P. J. Montero, J. A. Font, and V. Mewes, Phys. Rev. D 92, 083001 (2015).

[38] N. Sanchis-Gual, J. C. Degollado, P. Izquierdo, J. A. Font, and P. J. Montero, Phys. Rev. D 94, 043004 (2016).

[39] N. Sanchis-Gual, J. C. Degollado, P. J. Montero, J. A. Font, and C. Herdeiro, Phys. Rev. Lett. 116, 141101 (2016).

[40] S. Detweiler, Phys. Rev. D 22, 2323 (1980).

[41] S. R. Dolan, Phys. Rev. D 76, 084001 (2007), arXiv:0705.2880 [gr-qc].

[42] R. Brito, V. Cardoso, and P. Pani, Superradiance, Vol. 906 (2015).

[43] W. E. East and F. Pretorius, Physical review letters 119, 041101 (2017).

[44] W. E. East, Physical Review D 96, 024004 (2017).

[45] W. E. East, F. M. Ramazanoğlu, and F. Pretorius, Phys. Rev. D 89, 061503 (2014), arXiv:1312.4529 [gr$\mathrm{qc}]$.

[46] H. Okawa, Classical and Quantum Gravity 32, 214003
(2015).

[47] C. A. Herdeiro and E. Radu, Physical Review Letters 112, 221101 (2014).

[48] C. Herdeiro and E. Radu, Class. Quant. Grav. 32, 144001 (2015), arXiv:1501.04319 [gr-qc].

[49] A. Arvanitaki, M. Baryakhtar, S. Dimopoulos, S. Dubovsky, and R. Lasenby, Phys. Rev. D 95, 043001 (2017), arXiv:1604.03958 [hep-ph].

[50] R. Brito, S. Ghosh, E. Barausse, E. Berti, V. Cardoso, I. Dvorkin, A. Klein, and P. Pani, Phys. Rev. D 96, 064050 (2017), arXiv:1706.06311 [gr-qc].

[51] Q. Yang, L.-W. Ji, B. Hu, Z.-J. Cao, and R.G. Cai, Res. Astron. Astrophys. 18, 065 (2018), arXiv:1706.00678 [gr-qc].

[52] C. Palomba, S. D'Antonio, P. Astone, S. Frasca, G. Intini, I. La Rosa, P. Leaci, S. Mastrogiovanni, A. L. Miller, F. Muciaccia, O. J. Piccinni, L. Rei, and F. Simula, Phys. Rev. Lett. 123, 171101 (2019), arXiv:1909.08854 [astro-ph.HE].

[53] D. Baumann, H. S. Chia, and R. A. Porto, Phys. Rev. D 99, 044001 (2019), arXiv:1804.03208 [gr-qc].

[54] L. Sun, R. Brito, and M. Isi, Phys. Rev. D 101, 063020 (2020).

[55] N. Siemonsen and W. E. East, Phys. Rev. D 101, 024019 (2020).

[56] S. Ghosh, E. Berti, R. Brito, and M. Richartz, Phys. Rev. D 99, 104030 (2019).

[57] M. Isi, L. Sun, R. Brito, and A. Melatos, Phys. Rev. D 99, 084042 (2019).

[58] K. K. Y. Ng, M. Isi, C.-J. Haster, and S. Vitale, "Multiband gravitational-wave searches for ultralight bosons," (2020), arXiv:2007.12793 [gr-qc].

[59] J. C. Bustillo, N. Sanchis-Gual, A. Torres-Forné, J. A. Font, A. Vajpeyi, R. Smith, C. Herdeiro, E. Radu, and S. H. Leong, (2020), arXiv:2009.05376 [gr-qc].

[60] H. Okawa, H. Witek, and V. Cardoso, Phys. Rev. D 89, 104032 (2014).

[61] J. Barranco, A. Bernal, J. C. Degollado, A. DiezTejedor, M. Megevand, M. Alcubierre, D. Nunez, and O. Sarbach, Phys. Rev. Lett. 109, 081102 (2012), arXiv:1207.2153 [gr-qc].

[62] J. Barranco, A. Bernal, J. C. Degollado, A. DiezTejedor, M. Megevand, M. Alcubierre, D. Núñez, and O. Sarbach, Phys. Rev. D 89, 083006 (2014), arXiv:1312.5808 [gr-qc].

[63] J. Barranco, A. Bernal, J. C. Degollado, A. DiezTejedor, M. Megevand, M. Alcubierre, D. Nunez, and O. Sarbach, Phys. Rev. D 84, 083008 (2011), arXiv:1108.0931 [gr-qc].

[64] T. Nakamura, K. Oohara, and Y. Kojima, Prog. Theor. Phys. Suppl. 3, 1 (1987).

[65] M. Shibata and T. Nakamura, Phys. Rev. D 52, 5428 (1995).

[66] T. W. Baumgarte and S. L. Shapiro, Phys. Rev. D 59, 024007 (1998).

[67] M. Alcubierre, G. Allen, B. Brügmann, T. Dramlitsch, J. A. Font, P. Papadopoulos, E. Seidel, N. Stergioulas, W.-M. Suen, and R. Takahashi, Phys. Rev. D62, 044034 (2000), arXiv:gr-qc/0003071 [gr-qc].

[68] M. Alcubierre, B. Brügmann, P. Diener, M. Koppitz, D. Pollney, E. Seidel, and R. Takahashi, Phys. Rev. D67, 084023 (2003), arXiv:gr-qc/0206072 [gr-qc].

[69] F. Löffler, J. Faber, E. Bentivegna, T. Bode, P. Diener, R. Haas, I. Hinder, B. C. Mundim, C. D. Ott, E. Schnet- 
ter, G. Allen, M. Campanelli, and P. Laguna, Class. Quantum Grav. 29, 115001 (2012), arXiv:1111.3344 [grqc].

[70] EinsteinToolkit, "Einstein Toolkit: Open software for relativistic astrophysics (ET_2019_03 "proca", released 2019-03-29)," .

[71] M. Zilhão and F. Löffler, Int.J.Mod.Phys. A28, 1340014 (2013), arXiv:1305.5299 [gr-qc].

[72] E. Schnetter, S. H. Hawley, and I. Hawke, Class. Quant. Grav. 21, 1465 (2004), arXiv:gr-qc/0310042.

[73] Carpet, http://www. carpetcode.org/, Carpet: Adaptive Mesh Refinement for the Cactus Framework.

[74] J. Thornburg, Class. Quant. Grav. 21, 743 (2004), arXiv:gr-qc/0306056.

[75] J. Thornburg, Phys. Rev. D 54, 4899 (1996), arXiv:grqc/9508014.

[76] J. D. Brown, P. Diener, O. Sarbach, E. Schnetter, and M. Tiglio, Phys. Rev. D 79, 044023 (2009), arXiv:0809.3533 [gr-qc].

[77] H. Witek, M. Zilhao, G. Ficarra, and M. Elley, "Canuda: a public numerical relativity library to probe fundamental physics," (2020).

[78] M. Zilhao, H. Witek, and V. Cardoso, Class. Quant. Grav. 32, 234003 (2015), arXiv:1505.00797 [gr-qc].

[79] N. Sanchis-Gual, F. Di Giovanni, M. Zilhão, C. Herdeiro, P. Cerdá-Durán, J. Font, and E. Radu, Phys. Rev. Lett. 123, 221101 (2019), arXiv:1907.12565 [gr-qc].

[80] F. Di Giovanni, N. Sanchis-Gual, P. Cerdá-Durán, M. Zilhão, C. Herdeiro, J. A. Font, and E. Radu, Phys. Rev. D 102, 124009 (2020), arXiv:2010.05845 [grqc].

[81] J. M. Bowen and J. W. York, Phys. Rev. D21, 2047 (1980).

[82] J. York, in Frontiers in Numerical Relativity, edited by C. Evans, L. Finn, and D. Hobill (Cambridge University Press, Cambridge, England, 1989) pp. 89-109.

[83] B. Bruegmann et al., (2006), gr-qc/0610128.

[84] M. Hannam, S. Husa, D. Pollney, B. Brugmann, and N. O'Murchadha, Phys. Rev. Lett. 99, 241102 (2007), gr-qc/0606099.

[85] M. Ansorg, B. Brügmann, and W. Tichy, Phys. Rev. D 70, 064011 (2004), arXiv:gr-qc/0404056.

[86] J. G. Baker, M. Campanelli, C. O. Lousto, and R. Takahashi, Phys. Rev. D65, 124012 (2002), arXiv:astroph/0202469 [astro-ph].

[87] G. B. Cook, Phys. Rev. D50, 5025 (1994), arXiv:grqc/9404043.

[88] E. Newman and R. Penrose, J. Math. Phys. 3, 566 (1962).

[89] Gravitational Wave postprocessing tool pyGWAnalysis: "svn.einsteintoolkit.org/pyGWAnalysis/trunk", http: //einsteintoolkit.org/.
[90] J. Aasi et al. (LIGO Scientific, VIRGO), Phys. Rev. D 89, 102006 (2014), arXiv:1403.5306 [gr-qc].

[91] D. Talukder, E. Thrane, S. Bose, and T. Regimbau, Phys. Rev. D 89, 123008 (2014), arXiv:1404.4025 [grqc].

[92] S. Bose, S. Dhurandhar, A. Gupta, and A. Lundgren, Phys. Rev. D 94, 122004 (2016).

[93] G. Ashton, M. Hübner, P. D. Lasky, C. Talbot, K. Ackley, S. Biscoveanu, Q. Chu, A. Divakarla, P. J. Easter, B. Goncharov, F. Hernandez Vivanco, J. Harms, M. E. Lower, G. D. Meadors, D. Melchor, E. Payne, M. D. Pitkin, J. Powell, N. Sarin, R. J. E. Smith, and E. Thrane, ApJS 241, 27 (2019), arXiv:1811.02042 [astro-ph.IM].

[94] J. S. Speagle, MNRAS 493, 3132 (2020), arXiv:1904.02180 [astro-ph.IM].

[95] J. Skilling, in American Institute of Physics Conference Series, American Institute of Physics Conference Series, Vol. 735, edited by R. Fischer, R. Preuss, and U. V. Toussaint (2004) pp. 395-405.

[96] S. Husa, S. Khan, M. Hannam, M. Pürrer, F. Ohme, X. J. Forteza, and A. Bohé, Phys. Rev. D 93, 044006 (2016).

[97] S. Khan, S. Husa, M. Hannam, F. Ohme, M. Pürrer, X. J. Forteza, and A. Bohé, Phys. Rev. D 93, 044007 (2016).

[98] A. L. anticipated Sensitivity curves, https://dcc. ligo.org/LIGO-T0900288/public.

[99] D. Talukder, S. Bose, S. Caudill, and P. T. Baker, Phys. Rev. D 88, 122002 (2013).

[100] O. Dreyer, B. Krishnan, D. Shoemaker, and E. Schnetter, Phys. Rev. D67, 024018 (2003), arXiv:grqc/0206008.

[101] A. Ashtekar and B. Krishnan, Living Rev. Rel. 7, 10 (2004), arXiv:gr-qc/0407042.

[102] E. Schnetter, B. Krishnan, and F. Beyer, Phys. Rev. D74, 024028 (2006), arXiv:gr-qc/0604015.

[103] A. Gupta, B. Krishnan, A. Nielsen, and E. Schnetter, Phys. Rev. D97, 084028 (2018), arXiv:1801.07048 [grqc].

[104] V. Prasad, A. Gupta, S. Bose, B. Krishnan, and E. Schnetter, Phys. Rev. Lett. 125, 121101 (2020), arXiv:2003.06215 [gr-qc].

[105] P. Mourier, X. Jimenez-Forteza, D. Pook-Kolb, B. Krishnan, and E. Schnetter, (2020), arXiv:2010.15186 [gr-qc].

[106] A. Nitz, I. Harry, D. Brown, C. M. Biwer, J. Willis, T. D. Canton, C. Capano, L. Pekowsky, T. Dent, A. R. Williamson, G. S. Davies, S. De, M. Cabero, B. Machenschalk, P. Kumar, S. Reyes, D. Macleod, F. Pannarale, dfinstad, T. Massinger, M. Tápai, L. Singer, S. Khan, S. Fairhurst, S. Kumar, A. Nielsen, SSingh087, shasvath, I. Dorrington, and B. U. V. Gadre, "gwastro/pycbc: Pycbc release v1.16.7," (2020). 